Reprod. Nutr. Dévelop., 1988, 28 (1), 183-184.

\title{
Cinétique d'ingestion lors du grand repas chez le mouton : influence du temps d'accès au foin et de son ingestibilité
}

R. BAUMONT

Laboratoire des Aliments,

I.N.R.A., Theix, 63122 Ceyrat, France.

Summary. The food intake of sheep fed hay during the main meals has been adjusted to a mono-exponential model in order to determine the initial and final eating rates characterizing the hunger and satiety states.

L'évolution de la quantité ingérée cumulée au cours du grand repas peut être ajustée très précisément, chez la vache, à un modèle monomoléculaire (Faverdin, 1985). Cela permet de connaître la vitesse d'ingestion instantanée et de calculer les vitesses d'ingestion initiale et finale afin de préciser les états de faim et de satiété. Un tel ajustement a été effectué pour 240 grands repas observés chez 6 moutons.

Matériel et méthodes. L'enregistrement continu sur micro-ordinateur de la quantité ingérée est obtenu par un système d'auges sur jauges de contrainte. Six moutons (mâles castrés, 2-3 ans) alimentés à volonté deux fois par jour, à $8 \mathrm{~h}$ et $16 \mathrm{~h}$, ont reçu un foin de prairie successivement en accès continu, puis en accès limité à deux fois $1 \mathrm{~h} 30$. Ensuite, ils ont reçu un foin de luzerne, nettement plus ingestible, selon le même schéma.

Les cinétiques d'ingestion lors des grands repas suivant les distributions ont été regroupées par animal et par période sur 5 jours consécutifs laprès adaptation de 15 jours au foin et de 8 jours au temps d'accès limité) en distiguant les repas du matin et du soir. Les données ont été ajustées par la méthode des moindres carrés non linéaire au modèle suivant : $\mathrm{Q} \mid(T)=A(1-\exp (-\mathrm{bT}))$ avec $\mathrm{QI}(\mathrm{T})=$ quantité ingérée en $\mathrm{g}$ de $\mathrm{MS}$ et $\mathrm{T}=$ temps en minutes.

Résultats et discussion. L'ajustement des résultats au modèle est satisfaisant (coefficient de détermination compris entre 0,75 et 0,98 ) et l'écart-type résiduel est de l'ordre de $50 \mathrm{~g}$ de MS. La valeur de l'asymptote $A$ est liée positivement à la quantité ingérée ainsi qu'à la durée d'ingestion. Le paramètre $b$, qui est positivement corrélé avec le rapport des vitesses d'ingestion initiale et finale, correspond donc bien à une notion de freinage. Un tel modèle avec une vitesse continuellement décroissante lobservée par Forbes et al., 1972, chez le mouton et par Suzuki et al., 1973, chez la vache) est donc satisfaisant pour l'étude des cinétiques d'ingestion lors du grand repas chez le ruminant.

Lorsque l'ingestibilité du foin est accrue, les vitesses d'ingestion initiale et finale augmentent (tabl. 1). La vitesse initiale d'ingestion, corrélée positivement avec la quantité ingérée lors du grand repas (QIGR), semble donc caractériser l'appétit de l'animal pour le fourrage (cf. Kenney et Black, 1984). L'ingestibilité du foin ne modifie pas significativement la vitesse finale relative, ni le rapport (AQIGR)/A. Ces deux critères caractérisent ainsi l'état de satiété chez le mouton en accord avec les données obtenues chez la vache (Faverdin, 1985).

Lorsque le temps d'accès est limité à deux fois 1 h 30 , les quantités ingérées sont maintenues avec le foin de prairie, mais non avec le foin de luzerne. 
TABL. 1. - Effets de l'ingestibilité du foin et du temps d'accès sur les quantités ingérées, les caractéristiques des grands repas et les paramètres de leur modélisation sur un lot de six moutons $(\mathrm{n}=48$ ).

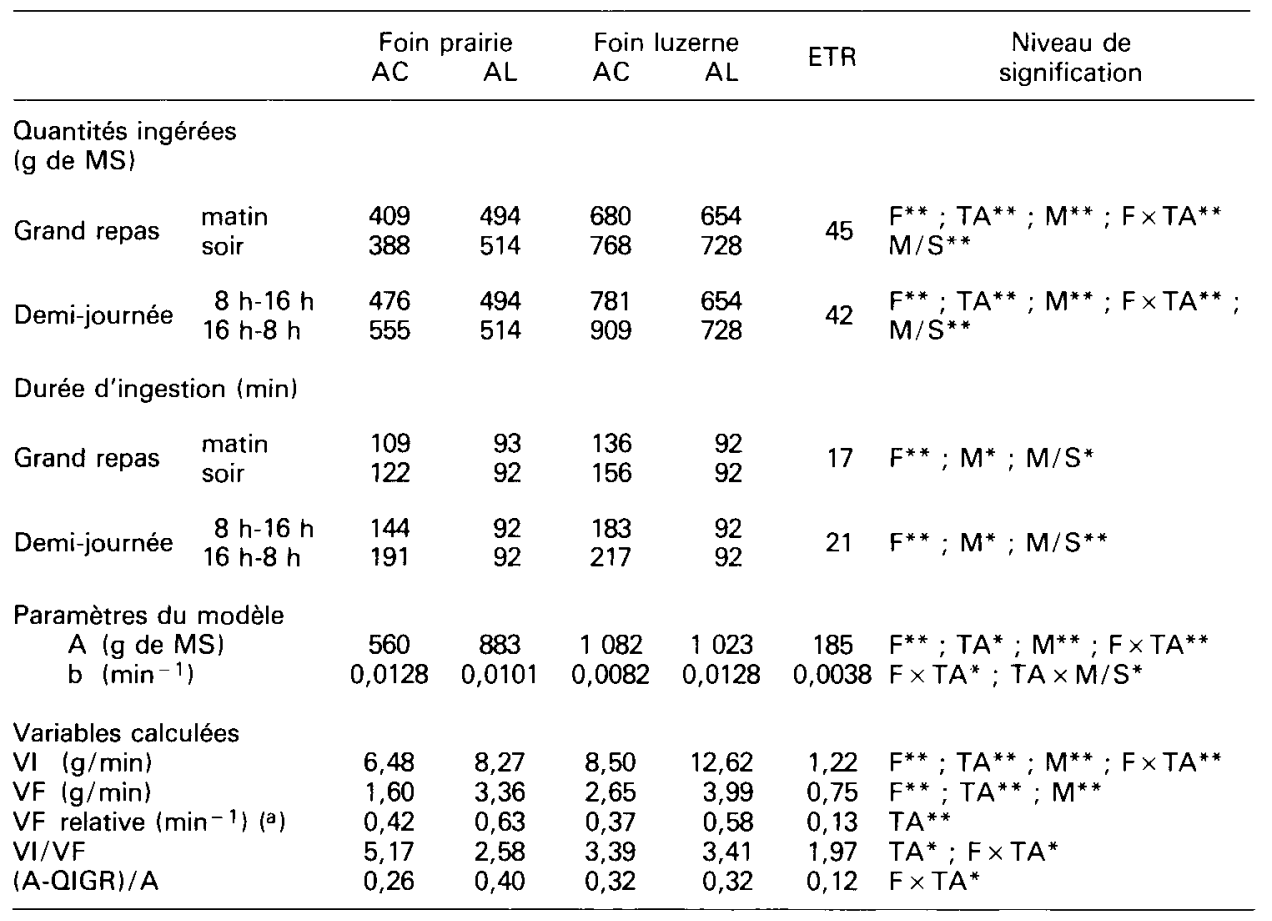

$A C=$ accès continu. $A L=$ accès limité. $Q I G R=$ quantité ingérée par grand repas. $V I=$ vitesse initiale. $V F=$ vitesse finale. $F=$ foin. $T A=$ temps d'accès $A C$ ou $A L . M=$ mouton. $M / S=$ matin/soir. $F \times T A=$ interaction foin $\times$ temps d'accès. (a) (vitesse finale/QIGR) $\times 100 .{ }^{*} P<0,05 ;{ }^{*} P<0,01$. ETR = écart-type résiduel.

L'accroissement des vitesses initiale et finale d'ingestion peut être interprété par un état de faim accru et un entraînement à la consommation accélérée. Avec le foin de prairie, la QIGR est plus élevée qu'en accès continu, d'où une diminution de la constante de freinage $b$ ainsi que du rapport des vitesses d'ingestion initiale et finale.

L'augmentation de la quantité ingérée le soir est la conséquence d'une durée d'ingestion accrue et n'a pas d'effet sur les paramètres du modèle.

En conclusion, les paramètres obtenus par l'ajustement des cinétiques d'ingestion lors du grand repas à un modèle monomoléculaire, permettent de mieux caractériser chez le mouton, comme chez les bovins, les états de faim et de satiété.

Faverdin P., 1985. Régulation de l'ingestion des vaches laitières en début de lactation. Th. Doct. Ing. INAPG.

Forbes J. M., Wright J. A., Bannister A., 1972. Anim. Prod., 15, 211-214.

Kenney P. A., Black J. L., 1984. Aust. J. Agric. Res., 35, 839-843.

Suzuki S., Shinde Y., Hidari H., 1973. Jap. J. zootech. Sci., 44, 181-187. 